\title{
Forecast for cosmological parameter estimation with gravitational-wave standard siren observation from the Cosmic Explorer
}

\author{
Shang-Jie Jin, ${ }^{a}$ Dong-Ze He, ${ }^{a}$ Yidong $\mathbf{X u},{ }^{b, 1}$ Jing-Fei Zhang, ${ }^{a}$ Xin \\ Zhang $^{a, c, d, 1}$
}

${ }^{a}$ Department of Physics, College of Sciences, Northeastern University, Shenyang 110819, China

${ }^{b}$ Key Laboratory for Computational Astrophysics, National Astronomical Observatories, Chinese Academy of Sciences, Beijing 100101, China

${ }^{c}$ Ministry of Education's Key Laboratory of Data Analytics and Optimization for Smart Industry, Northeastern University, Shenyang 110819, China

${ }^{d}$ Center for High Energy Physics, Peking University, Beijing 100080, China

E-mail: 850632821@qq.com, hedongze1992@163.com, xuyd@nao.cas.cn,

jfzhang@mail.neu.edu.cn, zhangxin@mail.neu.edu.cn

\begin{abstract}
The third-generation ground-based gravitational-wave (GW) detector, Cosmic Explorer (CE), is scheduled to start its observation in the 2030s. In this paper, we make a forecast for cosmological parameter estimation with gravitational-wave standard siren observation from the CE. We use the simulated GW standard siren data of CE to constrain the $\Lambda \mathrm{CDM}, w \mathrm{CDM}$ and CPL models. We combine the simulated GW data with the current cosmological electromagnetic observations including the latest cosmic microwave background anisotropies data from Planck, the optical baryon acoustic oscillation measurements, and the type Ia supernovae observation (Pantheon compilation) to do the analysis. We find that the future standard siren observation from $\mathrm{CE}$ will improve the cosmological parameter estimation to a great extent, since the future GW standard siren data can well break the degeneracies generated by the optical observations between various cosmological parameters. We also find that the CE's constraining capability on the cosmological parameters is slightly better than that of the same-type GW detector, the Einstein Telescope. In addition, the synergy between the GW standard siren observation from $\mathrm{CE}$ and the $21 \mathrm{~cm}$ emission observation from SKA is also discussed.
\end{abstract}

\footnotetext{
${ }^{1}$ Corresponding author.
} 


\section{Contents}

1 Introduction $\quad 1$

2 Gravitational wave standard sirens 3

3 Method and data $\quad 4$

3.1 Data simulation for the GW standard sirens 4

3.2 Current mainstream optical cosmological probes 6

$\begin{array}{lll}3.3 & \text { Combined constraints } & 7\end{array}$

$\begin{array}{lll}4 & \text { Results } & 7\end{array}$

5 Some discussions $\quad 12$

5.1 Comparison of CE and ET 12

$\begin{array}{lll}5.2 & \text { Synergy with SKA } & 12\end{array}$

6 Conclusion 13

\section{Introduction}

Nowadays, the study of cosmology has entered the era of precision cosmology, and some great achievements have been made. For example, the accelerated expansion of the universe has been discovered [1,2]; some cosmological parameters have been precisely measured [3]; and in particular, a standard model of cosmology, which refers to the base six-parameter $\Lambda$ cold dark matter model (usually abbreviated as $\Lambda$ CDM model), has been established. In this model, the cosmological constant (vacuum energy) $\Lambda$ with the equation of state (EoS) $w=-1$ serves as dark energy (DE) responsible for the late-time cosmic acceleration. The measurements of cosmic microwave background ( $\mathrm{CMB}$ ) anisotropies from Planck satellite mission [3] have newly constrained the six primary parameters within the $\Lambda \mathrm{CDM}$ model with unprecedented precision. Notwithstanding, some parameter degeneracies still exist in the standard model. Thus, it is far-fetched to take the $\Lambda$ CDM model as the eventual cosmic scenario. However, when the $\Lambda \mathrm{CDM}$ model is extended to include some new physics, e.g., a general dynamical dark energy, massive neutrinos, dark radiation, primordial gravitational waves, etc., the newly introduced parameters will severely degenerate with other parameters when the CMB alone data are used to constrain the extended models. Therefore, some other cosmological probes exploring the late-time universe are needed to be combined with the CMB data to break the parameter degeneracies.

The cosmological probes in light of the optical observations have been developed for many years. Besides the CMB observation, the mainstream cosmological probes mainly include, e.g., type Ia supernovae (SN), baryon acoustic oscillations (BAO), redshift space distortions (RSD), weak lensing, galaxy cluster counts, etc., which are all based on the observations of electromagnetic waves. In the future 10-15 years, these probes will be further greatly developed, and the fourth-generation DE programs, e.g., DESI [4], Euclid [5], and LSST [6] will be implemented. However, other than those optical (or near-infrared) observations, some other new types of the cosmological probe should also be developed. The most promising 
new-type cosmological probes include the observations of gravitational waves (GWs) and of $21 \mathrm{~cm}$ radio waves.

The first detection of the GWs from the binary neutron star (BNS) merger (GW170817 [7]; actually, the detection was only for the inspiral of the BNS) was rather meaningful, because it initiated the new era of multi-messenger astronomy. For this event, not only the GWs but also the electromagnetic waves in various bands [8] were observed. It is well-known that GWs can serve as standard sirens since the waveform of GWs carries the information of luminosity distance to the source. Therefore, the multi-messenger observation of the GW standard sirens can provide us with both the information of distance and redshift, which can be used to study cosmology. For example, the event of GW170817 has been used to independently measure the Hubble constant [9]. The main advantage of such a standard siren method is that it avoids using the cosmic distance ladder. But due to the fact that we now have only one actual standard siren data point, the error of the Hubble constant measurement is still large (around 15\%), and thus it still cannot make an arbitration for the Hubble tension. Of course, with the accumulation of observed standard siren events, in the future the Hubble tension will be definitely resolved.

In the future, the GW standard sirens would also be developed into a powerful cosmological probe. The third-generation ground-based GW detectors have been proposed, e.g., the Einstein Telescope (ET) [10] in the Europe and the Cosmic Explorer (CE) [11] in the United States. The detection ability of the third-generation ground-based GW detectors is much better than that of the second-generation ones [12-14]. Using ET or CE, one can observe much more BNS merger events up to much higher redshifts, and thus they can play an important role in the cosmological parameter estimation and related issues in the future.

Recently, some issues concerning the forecasts for future cosmological parameter estimation based on the ET have been discussed [15-21]. It is found that the GW standard siren observation from the ET would play a rather significant role in the measurement of cosmological parameters because the standard sirens can break the parameter degeneracies generated by the other cosmological observations (CMB combined with other lower-redshift observations). This is mainly because GW observation can provide a measurement of the absolute luminosity distance (not the relative one). In addition, the space-based GW observatories have also been proposed and are being built, e.g., the Laser Interferometer Space Antenna (LISA), TianQin and Taiji projects [22-30]. The space-based GW detectors aim at detecting low-frequency GWs mainly generated from supermassive black hole coalescence and extreme mass ratio inspiral. The GW standard siren observations from the space-based GW observatories can also improve the cosmological parameter estimation to some extent $[31,32]$, although there are still some uncertainties in the observations and data simulation.

In this paper, we will make a forecast for the cosmological parameter estimation using the GW standard siren observation from the CE. We will investigate what role the CE's GW standard siren observation would play in the future cosmological parameter measurement. We will focus the discussions on the cosmological parameter constraint capability of the $\mathrm{CE}$, in particular for the cases considering the combination with the optical cosmological probes. What's more, since both CE and ET belong to the third-generation ground-based GW detectors, in this work we also make a comparison for the promotion effects on the parameter estimation from $\mathrm{CE}$ and $\mathrm{ET}$.

We consider three most typical DE cosmological models in this work, namely the $\Lambda$ CDM model, the $w \mathrm{CDM}$ model, and the Chevalliear-Polarski-Linder (CPL) model. We will use the simulated standard siren data from the $\mathrm{CE}$ to constrain the cosmological parameters in these 
three DE models. Of course, the main task of this work is to investigate what role the standard sirens from the CE will play in combination with other cosmological observations. Therefore, we will combine the standard sirens with the current mainstream optical observations, i.e., the $\mathrm{CMB}+\mathrm{BAO}+\mathrm{SN}$ data, to investigate how the standard sirens from the $\mathrm{CE}$ would break the cosmological parameter degeneracies generated by the optical observations.

In addition to the GW standard sirens, the neutral hydrogen (HI) $21 \mathrm{~cm}$ emission observation is another important non-optical cosmological probe. In the future, the $21 \mathrm{~cm}$ radio observation by e.g. the Square Kilometre Array (SKA) [33] will provide the HI power spectrum and the related BAO as well as RSD measurements, which will also play an important role in the future cosmological parameter estimation (see, e.g., the recent studies [34-38] and brief review [39]). Therefore, in this work, we will also discuss the synergy of the GW standard siren observation from the $\mathrm{CE}$ and the $\mathrm{HI} 21 \mathrm{~cm}$ emission observation from the SKA.

\section{Gravitational wave standard sirens}

The most important advantage of the GW standard siren observation, compared with the current optical cosmological observations, is that the GW signals can provide the measurement of the absolute luminosity distance. Since the GW amplitude depends on the luminosity distance, we can naturally extract the information of luminosity distance from the analysis of the waveform.

For a Friedmann-Robertson-Walker universe, the line element reads

$$
d s^{2}=-d t^{2}+a^{2}(t)\left[\frac{d r^{2}}{1-K r^{2}}+r^{2}\left(d \theta^{2}+\sin ^{2} \theta d \phi^{2}\right)\right],
$$

where $t$ is the cosmic time, $a(t)$ is the scale factor, and $K=+1,-1$, and 0 correspond to closed, open, and flat universes, respectively. We set $G=c=1$ and $K=0$ throughout this paper. In a flat universe, the luminosity distance $d_{L}$ can be written as

$$
d_{L}(z)=\frac{(1+z)}{H_{0}} \int_{0}^{z} \frac{d z^{\prime}}{E\left(z^{\prime}\right)},
$$

where $E(z) \equiv H(z) / H_{0}$. For different DE models, the forms of $E(z)$ can be found in, e.g., ref. [40].

Considering the transverse-traceless (TT) gauge, the strain $h(t)$ in the GW interferometers is given by [15]

$$
h(t)=F_{+}(\theta, \phi, \psi) h_{+}(t)+F_{\times}(\theta, \phi, \psi) h_{\times}(t),
$$

where $F_{+, \times}$are the antenna pattern functions, $\psi$ is the polarization angle, and $(\theta, \phi)$ are angles describing the location of the source in the sky, relative to the detector. $h_{+}=h_{x x}=$ $-h_{y y}$, and $h_{\times}=h_{x y}=h_{y x}$, which are the two independent components of the GW's tensor $h_{\alpha \beta}$ in the TT gauge. Here, the antenna pattern functions of CE [41] are

$$
\begin{aligned}
& F_{+}(\theta, \phi, \psi)=\frac{1}{2}\left(1+\cos ^{2}(\theta)\right) \cos (2 \phi) \cos (2 \psi)-\cos (\theta) \sin (2 \phi) \sin (2 \psi), \\
& F_{\times}(\theta, \phi, \psi)=\frac{1}{2}\left(1+\cos ^{2}(\theta)\right) \cos (2 \phi) \sin (2 \psi)+\cos (\theta) \sin (2 \phi) \cos (2 \psi) .
\end{aligned}
$$


We also wish to make a direct comparison with the ET. For ET, the antenna pattern functions [42] become

$$
\begin{aligned}
& F_{+}^{(1)}(\theta, \phi, \psi)=\frac{\sqrt{3}}{2}\left[\frac{1}{2}\left(1+\cos ^{2}(\theta)\right) \cos (2 \phi) \cos (2 \psi)-\cos (\theta) \sin (2 \phi) \sin (2 \psi)\right], \\
& F_{\times}^{(1)}(\theta, \phi, \psi)=\frac{\sqrt{3}}{2}\left[\frac{1}{2}\left(1+\cos ^{2}(\theta)\right) \cos (2 \phi) \sin (2 \psi)+\cos (\theta) \sin (2 \phi) \cos (2 \psi)\right] .
\end{aligned}
$$

Since ET has three interferometers with $60^{\circ}$ inclined angles between each other, the other two antenna pattern functions are supposed to be $F_{+, \times}^{(2)}(\theta, \phi, \psi)=F_{+, \times}^{(1)}(\theta, \phi+2 \pi / 3, \psi)$ and $F_{+, \times}^{(3)}(\theta, \phi, \psi)=F_{+, \times}^{(1)}(\theta, \phi+4 \pi / 3, \psi)$, respectively.

Following refs. [42, 43], we also apply the stationary phase approximation to the computation of the Fourier transform $\mathcal{H}(f)$ of the time domain waveform $h(t)$,

$$
\mathcal{H}(f)=\mathcal{A} f^{-7 / 6} \exp \left[i\left(2 \pi f t_{0}-\pi / 4+2 \Psi(f / 2)-\varphi_{(2.0)}\right)\right],
$$

where the Fourier amplitude $\mathcal{A}$ is given by

$$
\mathcal{A}=\frac{1}{d_{L}} \sqrt{F_{+}^{2}\left(1+\cos ^{2}(\iota)\right)^{2}+4 F_{\times}^{2} \cos ^{2}(\iota)} \sqrt{5 \pi / 96} \pi^{-7 / 6} \mathcal{M}_{c}^{5 / 6},
$$

where $\mathcal{M}_{c}=M \eta^{3 / 5}$ is called "chirp mass", $M=m_{1}+m_{2}$ is the total mass of coalescing binary with component masses $m_{1}$ and $m_{2}, \eta=m_{1} m_{2} / M^{2}$ is the symmetric mass ratio. Here, it is necessary to point out that the observed chirp mass is related to the physical mass via $\mathcal{M}_{c, \text { obs }}=(1+z) \mathcal{M}_{c, \text { phys }}$ [15]. $\mathcal{M}_{c}$ in eq. (2.7) represents the observed chirp mass. The constant $t_{0}$ denotes the time of the merger. $\iota$ is the angle of inclination of the binary's orbital angular momentum with the line of sight. The definitions of the functions $\Psi$ and $\varphi_{(2.0)}$ can refer to refs. [42, 43]. Since it is expected that the short gamma ray bursts (SGRBs) are expected to be strongly beamed [45-47], the coincidence observations of SGRBs imply that the binaries are orientated nearly face on (i.e., $\iota \simeq 0$ ) and the maximal inclination is about $\iota=20^{\circ}$. Actually, averaging the Fisher matrix over the inclination $\iota$ and the polarization $\psi$ with the constraint $\iota<20^{\circ}$ is approximately the same as taking $\iota=0$ in the simulation [43]. Thus, we take $\iota=0$ in the calculation of the central values of the luminosity distances in the process of simulating GW sources.

\section{Method and data}

In this section, we shall introduce the method of simulating the GW standard siren data from the CE. In section 5, since we discuss the comparison of CE and ET, we also introduce the method of simulating the GW standard siren data from the ET. We first describe how to get the errors of the luminosity distances to the GW sources in the simulation of standard siren data. Then, we introduce the current mainstream optical cosmological probes utilized in this work. Finally, we will use the Markov-chain Monte Carlo (MCMC) approach to infer the posterior distributions of cosmological parameters.

\subsection{Data simulation for the GW standard sirens}

In this paper, we focus on the BNS systems and we assume that both neutron stars have mass of $m_{1}=m_{2}=1.4 M_{\odot}$, where $M_{\odot}$ is the solar mass. The first step for generating 


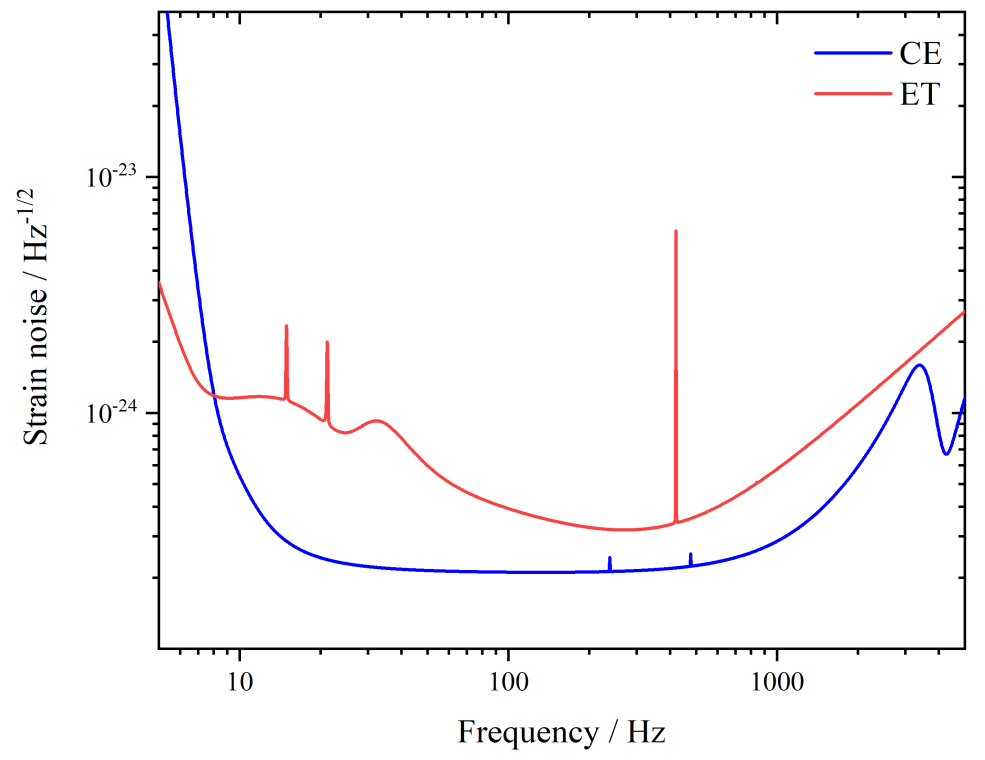

Figure 1. The sensitivity curves of $\mathrm{CE}$ and ET, of which the text data files can be found in ref. [50] and ref. [51], respectively.

GW data is to simulate the redshift distribution of the sources. Following refs. [15, 42], the redshift distribution of the sources takes the form

$$
P(z) \propto \frac{4 \pi d_{C}^{2}(z) R(z)}{H(z)(1+z)},
$$

where $d_{C}$ is the comoving distance, which is defined as $d_{C}(z) \equiv \int_{0}^{z} 1 / H\left(z^{\prime}\right) d z^{\prime}$, and $R(z)$ describes the time evolution of the burst rate and takes the form $[48,49]$

$$
R(z)= \begin{cases}1+2 z, & z \leq 1 \\ \frac{3}{4}(5-z), & 1<z<5 \\ 0, & z \geq 5\end{cases}
$$

The combined signal-to-noise ratio (SNR) for the network of $N(N=1$ for $\mathrm{CE}$, and $N=3$ for ET) independent interferometers is given by

$$
\rho=\sqrt{\sum_{i=1}^{N}\left(\rho^{(i)}\right)^{2}},
$$

where $\rho^{(i)}=\sqrt{\left\langle\mathcal{H}^{(i)}, \mathcal{H}^{(i)}\right\rangle}$. The inner product is defined as

$$
\langle a, b\rangle=4 \int_{f_{\text {lower }}}^{f_{\text {upper }}} \frac{\tilde{a}(f) \tilde{b}^{*}(f)+\tilde{a}^{*}(f) \tilde{b}(f)}{2} \frac{d f}{S_{h}(f)},
$$


where $\tilde{a}(f)$ and $\tilde{b}(f)$ are the Fourier transforms of the functions $a(t)$ and $b(t)$. Here, $f_{\text {lower }}$ is the lower cutoff frequency. The upper cutoff frequency is dictated by the last stable orbit (LSO) and it marks the end of the inspiral regime and the onset of the final merger. Here, $f_{\text {upper }}=2 f_{\mathrm{LSO}}$, where $f_{\mathrm{LSO}}=1 /\left(6^{3 / 2} 2 \pi M_{\mathrm{obs}}\right)$ is the orbit frequency at the LSO, and $M_{\mathrm{obs}}=(1+z) M_{\mathrm{phys}}$ is the observed total mass [42]. The performance of a GW detector is characterized by the one-side noise power spectral density (PSD) $S_{h}(f)$. For the noise PSD of $\mathrm{CE}$, we use the interpolation method to fit the CE stage 2 data in ref. [50]. While for ET, we take the noise PSD to be the same as described in ref. [42] and the SNR threshold is taken to be 8 for a detection.

Using the Fisher information matrix, we can get the instrumental error of $d_{L}$,

$$
\sigma_{d_{L}}^{\text {inst }} \simeq \sqrt{\left\langle\frac{\partial \mathcal{H}}{\partial d_{L}}, \frac{\partial \mathcal{H}}{\partial d_{L}}\right\rangle^{-1}} .
$$

Because $\mathcal{H} \propto d_{L}^{-1}$, we can get $\sigma_{d_{L}}^{\text {inst }} \simeq d_{L} / \rho$. When we simulate the GW sources, we take $\iota=0$ and add a factor 2 in front of the error [43]. More details can be found in ref. [15]. The instrumental error of $d_{L}$ can be written as

$$
\sigma_{d_{L}}^{\text {inst }} \simeq \frac{2 d_{L}}{\rho}
$$

Besides, the luminosity distance is affected by the weak lensing which will lead to an additional error of $d_{L}[42,52]$,

$$
\sigma_{d_{L}}^{\text {lens }} \simeq 0.05 z d_{L}
$$

Thus, the total error of $d_{L}$ is

$$
\begin{aligned}
\sigma_{d_{L}} & =\sqrt{\left(\sigma_{d_{L}}^{\text {inst }}\right)^{2}+\left(\sigma_{d_{L}}^{\text {lens }}\right)^{2}} \\
& =\sqrt{\left(\frac{2 d_{L}}{\rho}\right)^{2}+\left(0.05 z d_{L}\right)^{2}} .
\end{aligned}
$$

Now, we can simulate the measurement of the redshifts with the luminosity distances for the GW events of BNS mergers. More details can be found in ref. [15]. Different from ref. [20], we only consider the case of $1.4-1.4 M_{\odot}$ BNS merger. Then we can generate the GW events with their $z, d_{L}$ and $\sigma_{d_{L}}$. In this work, we simulate $1000 \mathrm{GW}$ standard siren events expected to be detected by the CE in the 2040s [53].

\subsection{Current mainstream optical cosmological probes}

The current mainstream optical cosmological probes considered in this work include $\mathrm{CMB}, \mathrm{BAO}$, and SN. For the CMB data, we use the "Planck distance priors" from the Planck 2018 results $[3,54]$. For the BAO data, we use the measurements from 6dFGS at $z_{\text {eff }}=0.106$ [55], SDSS-MGS at $z_{\text {eff }}=0.15$ [56], and BOSS-DR12 at $z_{\text {eff }}=0.38,0.51$, and 0.61 [57]. For the SN data, we use the latest sample from the Pantheon compilation [58]. For convenience, the data combination "CMB+BAO+SN" is also abbreviated as "CBS" in the following. 


\subsection{Combined constraints}

In order to constrain the cosmological parameters, we use the MCMC method to infer their posterior probability distributions. The total $\chi^{2}$ function of the combination of CMB, $\mathrm{BAO}$ and SN data is

$$
\chi_{\mathrm{tot}}^{2}=\chi_{\mathrm{CMB}}^{2}+\chi_{\mathrm{BAO}}^{2}+\chi_{\mathrm{SN}}^{2} .
$$

In this paper, we simulate $1000 \mathrm{GW}$ data points. For the GW data, its $\chi^{2}$ can be written as

$$
\chi_{\mathrm{GW}}^{2}=\sum_{i=1}^{1000}\left[\frac{\bar{d}_{L}^{i}-d_{L}\left(\bar{z}_{i} ; \vec{\Omega}\right)}{\bar{\sigma}_{d_{L}}^{i}}\right]^{2},
$$

where $\bar{z}_{i}, \bar{d}_{L}^{i}$, and $\bar{\sigma}_{d_{L}}^{i}$ are the $i$ th redshift, luminosity distance, and error of luminosity distance, respectively. $\vec{\Omega}$ denotes a set of cosmological parameters.

If we consider the combination of the conventional cosmological electromagnetic observations and the GW standard siren observation, the total $\chi^{2}$ function becomes

$$
\chi_{\mathrm{tot}}^{2}=\chi_{\mathrm{CMB}}^{2}+\chi_{\mathrm{BAO}}^{2}+\chi_{\mathrm{SN}}^{2}+\chi_{\mathrm{GW}}^{2} .
$$

\begin{tabular}{|c|c|c|c|c|c|c|c|c|c|}
\hline \multirow{2}{*}{$\frac{\text { Model }}{\text { Data }}$} & \multicolumn{3}{|c|}{$\Lambda \mathrm{CDM}$} & \multicolumn{3}{|c|}{$w \mathrm{CDM}$} & \multicolumn{3}{|c|}{$\mathrm{CPL}$} \\
\hline & CBS & GW & $\mathrm{CBS}+\mathrm{GW}$ & CBS & GW & $\mathrm{CBS}+\mathrm{GW}$ & CBS & GW & $\mathrm{CBS}+\mathrm{GW}$ \\
\hline$\Omega_{\mathrm{m}}$ & $0.3142_{-0.0050}^{+0.0050}$ & $0.3143_{-0.0062}^{+0.0062}$ & $0.3142_{-0.0022}^{+0.0022}$ & $0.3118_{-0.0077}^{+0.0077}$ & $0.312_{-0.012}^{+0.013}$ & $0.3115_{-0.0023}^{+0.0023}$ & $0.3120_{-0.0078}^{+0.0078}$ & $0.296_{-0.024}^{+0.065}$ & $0.3118_{-0.0043}^{+0.0043}$ \\
\hline$H_{0}$ & $67.58_{-0.33}^{+0.33}$ & $67.58_{-0.19}^{+0.19}$ & $67.58_{-0.14}^{+0.14}$ & $67.92_{-0.83}^{+0.83}$ & $67.93_{-0.34}^{+0.34}$ & $67.92_{-0.22}^{+0.22}$ & $67.92_{-0.84}^{+0.84}$ & $67.87_{-0.62}^{+0.71}$ & $67.93_{-0.37}^{+0.37}$ \\
\hline$w$ & - & - & - & $-1.015_{-0.032}^{+0.032}$ & $-1.020_{-0.058}^{+0.064}$ & ${ }_{3}^{4}-1.014_{-0.017}^{+0.017}$ & - & - & - \\
\hline$w_{0}$ & - & - & - & - & - & - & $-0.992_{-0.083}^{+0.083}$ & $-0.952_{-0.120}^{+0.089}$ & $9-0.993_{-0.050}^{+0.050}$ \\
\hline$w_{a}$ & - & - & - & - & - & - & $-0.098_{-0.280}^{+0.330}$ & $-0.19_{-0.60}^{+1.20}$ & $-0.09_{-0.15}^{+0.17}$ \\
\hline
\end{tabular}

Table 1. The best-fit values for the parameters in the $\Lambda$ CDM, $w$ CDM and CPL models by using the $\mathrm{CBS}, \mathrm{GW}$, and $\mathrm{CBS}+\mathrm{GW}$ data combinations. Here, CBS stands for $\mathrm{CMB}+\mathrm{BAO}+\mathrm{SN}$ and $H_{0}$ is in units of $\mathrm{km} \mathrm{s}^{-1} \mathrm{Mpc}^{-1}$.

\section{Results}

In this section, we shall report the constraint results for the cosmological parameters in the $\Lambda \mathrm{CDM}, w \mathrm{CDM}$, and CPL models. We constrain the considered cosmological models with $\mathrm{GW}, \mathrm{CMB}+\mathrm{BAO}+\mathrm{SN}$, and $\mathrm{CMB}+\mathrm{BAO}+\mathrm{SN}+\mathrm{GW}$ data combinations to complete our analysis. The constraint results are shown in figure 2 and summarized in tables 1-2. In figure 2, we display the two-dimensional posterior distribution contours for various model parameters constrained at $68 \%$ and $95 \%$ confidence level (C.L.). In tables 1-2, we exhibit the best-fit values with $1 \sigma$ errors quoted and the constraint errors and accuracies for the concerned parameters (i.e., $\Omega_{\mathrm{m}}, H_{0}, w, w_{0}$, and $w_{a}$ ). Note that, for a parameter $\xi$, we use $\sigma(\xi)$ and $\varepsilon(\xi)$ to denote its absolute and relative errors in the cosmological fit, respectively.

At first glance of figure 2, we can clearly find that the future GW observation from $\mathrm{CE}$ could significantly improve the constraints on almost all the parameters to some different extent; for more details, see also tables 1-2. Particularly, in the $\Omega_{m}-H_{0}$ plane for the $\Lambda$ CDM 


\begin{tabular}{|c|c|c|c|c|c|c|c|c|c|}
\hline & \multicolumn{3}{|c|}{$\Lambda \mathrm{CDM}$} & \multicolumn{3}{|c|}{$w \mathrm{CDM}$} & \multicolumn{3}{|c|}{$\mathrm{CPL}$} \\
\hline & CBS & GW & $\mathrm{CBS}+\mathrm{GW}$ & CBS & GW & $\mathrm{CBS}+\mathrm{GW}$ & CBS & GW & $\mathrm{CBS}+\mathrm{GW}$ \\
\hline$\sigma\left(\Omega_{\mathrm{m}}\right)$ & 0.0050 & 0.0062 & 0.0022 & 0.0077 & 0.0125 & 0.0023 & 0.0078 & 0.0445 & 0.0043 \\
\hline$\sigma\left(H_{0}\right)$ & 0.330 & 0.190 & 0.140 & 0.830 & 0.340 & 0.220 & 0.840 & 0.665 & 0.370 \\
\hline$\sigma(w)$ & - & - & - & 0.032 & 0.061 & 0.017 & - & - & - \\
\hline$\sigma\left(w_{0}\right)$ & - & - & - & - & - & - & 0.0830 & 0.1045 & 0.0500 \\
\hline$\sigma\left(w_{a}\right)$ & - & - & - & - & - & - & 0.305 & 0.900 & 0.160 \\
\hline$\varepsilon\left(\Omega_{\mathrm{m}}\right)$ & 0.0159 & 0.0197 & 0.0070 & 0.0247 & 0.0401 & 0.0074 & 0.0250 & 0.1503 & 0.0138 \\
\hline$\varepsilon\left(H_{0}\right)$ & 0.0049 & 0.0028 & 0.0021 & 0.0122 & 0.0050 & 0.0032 & 0.0124 & 0.0098 & 0.0054 \\
\hline$\varepsilon(w)$ & - & - & - & 0.0315 & 0.0598 & 0.0168 & - & - & - \\
\hline$\varepsilon\left(w_{0}\right)$ & - & - & - & - & - & - & 0.0837 & 0.1098 & 0.0504 \\
\hline
\end{tabular}

Table 2. Constraint errors and accuracies for the parameters in the $\Lambda \mathrm{CDM}, w \mathrm{CDM}$ and CPL models by using the $\mathrm{CBS}, \mathrm{GW}$, and $\mathrm{CBS}+\mathrm{GW}$ data combinations. Here, $\mathrm{CBS}$ stands for $\mathrm{CMB}+\mathrm{BAO}+\mathrm{SN}$.
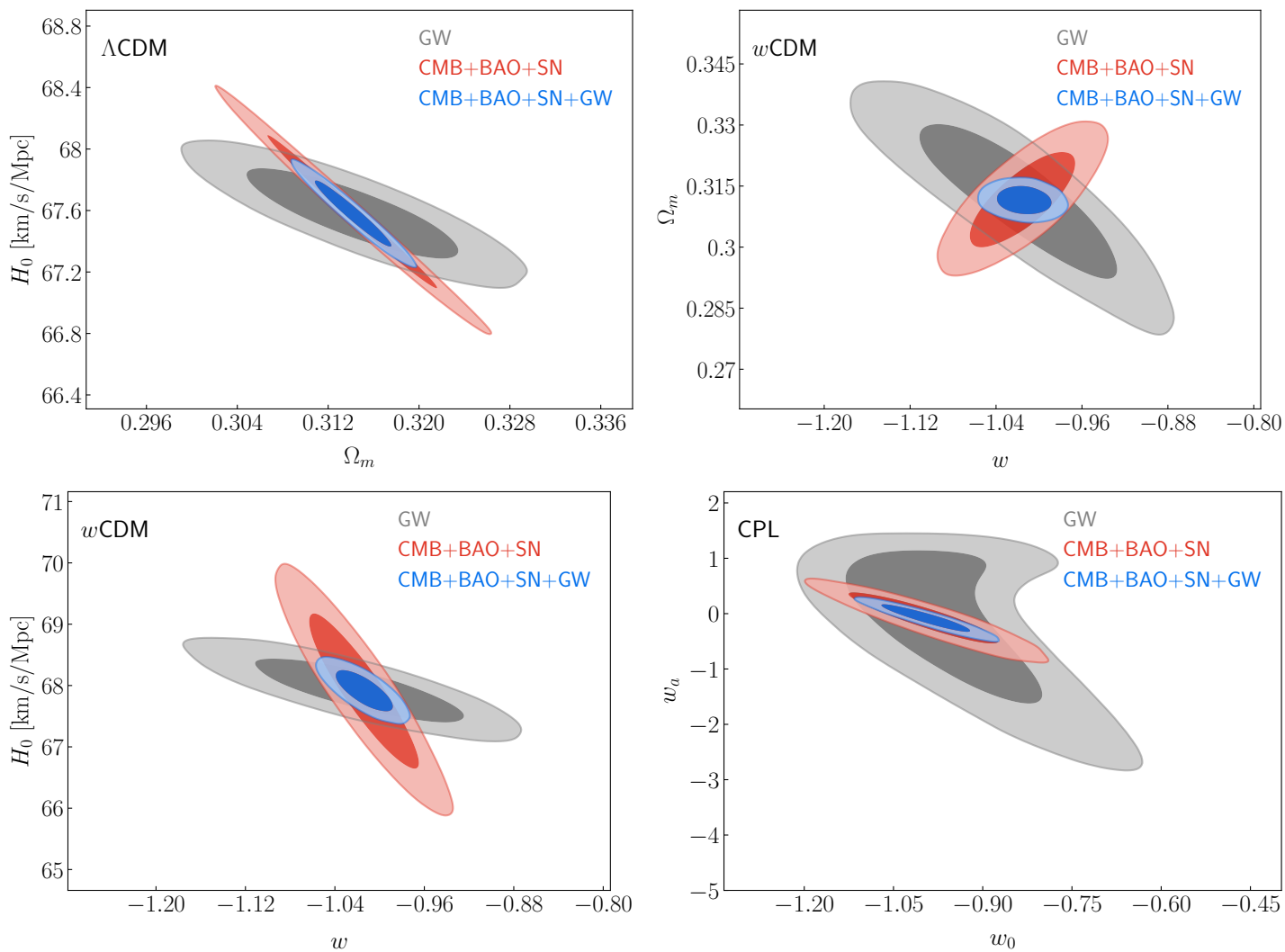

Figure 2. Constraints ( $68.3 \%$ and $95.4 \%$ confidence level) on the $\Lambda \mathrm{CDM}, w \mathrm{CDM}$, and CPL models by using the $\mathrm{GW}, \mathrm{CMB}+\mathrm{BAO}+\mathrm{SN}$, and $\mathrm{CMB}+\mathrm{BAO}+\mathrm{SN}+\mathrm{GW}$ data combinations. 

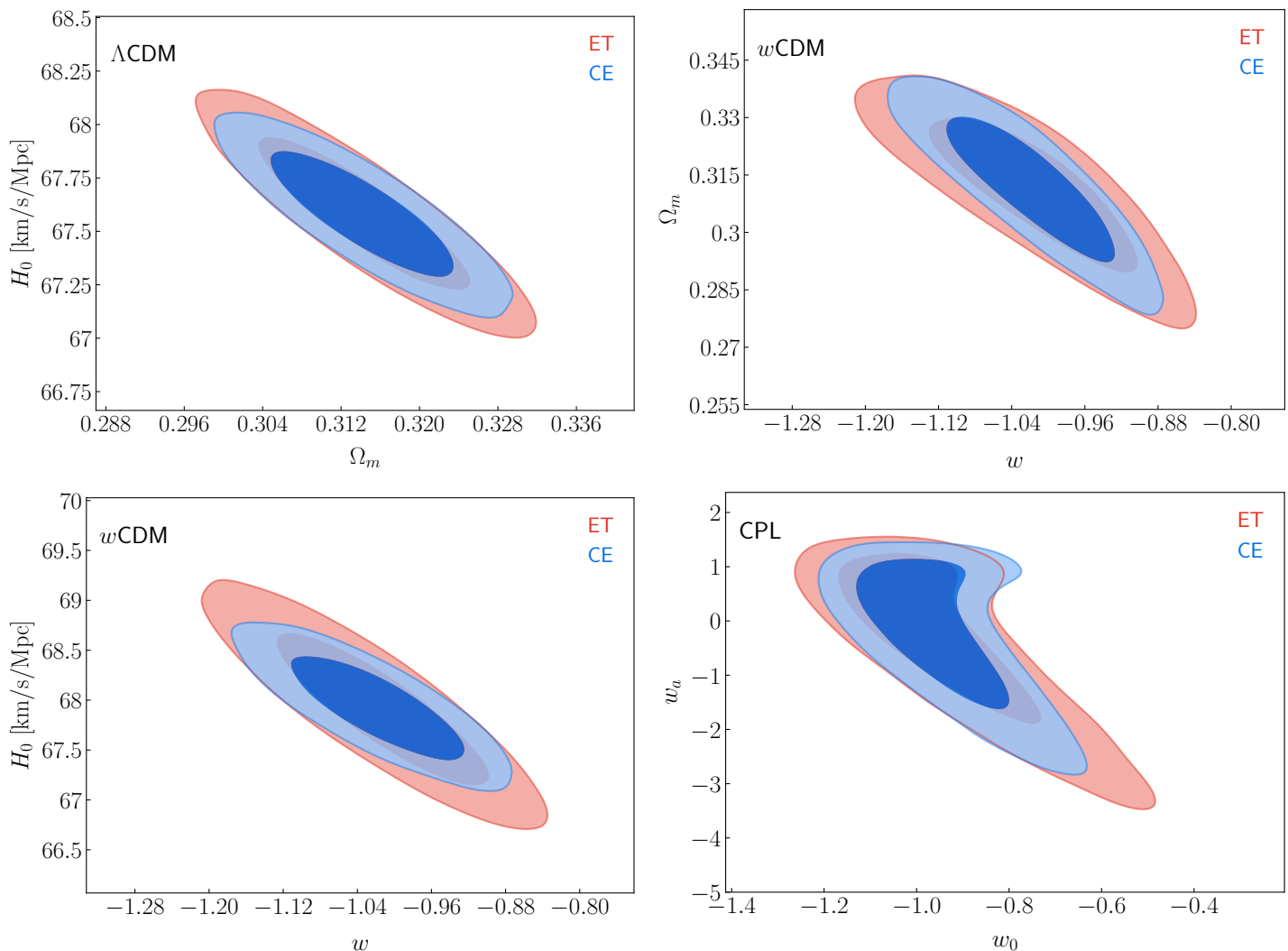

Figure 3. Constraints ( $68.3 \%$ and $95.4 \%$ confidence level) on the $\Lambda$ CDM, $w$ CDM, and CPL models by using the simulated data of ET and CE.

\begin{tabular}{ccccccccc}
\hline & \multicolumn{2}{c}{$\Lambda \mathrm{CDM}$} & & \multicolumn{2}{c}{$w \mathrm{CDM}$} & & \multicolumn{2}{c}{$\mathrm{CPL}$} \\
\cline { 2 - 3 } \cline { 8 - 9 } \cline { 8 - 9 } & $\mathrm{ET}$ & $\mathrm{CE}$ & & $\mathrm{ET}$ & $\mathrm{CE}$ & & $\mathrm{ET}$ & $\mathrm{CE}$ \\
\hline$\sigma\left(\Omega_{\mathrm{m}}\right)$ & 0.0071 & 0.0062 & & 0.0130 & 0.0125 & & 0.0450 & 0.0445 \\
$\sigma\left(H_{0}\right)$ & 0.240 & 0.190 & & 0.510 & 0.340 & & 0.940 & 0.665 \\
$\sigma(w)$ & - & - & & 0.075 & 0.061 & & - & - \\
$\sigma\left(w_{0}\right)$ & - & - & & - & - & & 0.135 & 0.1045 \\
$\sigma\left(w_{a}\right)$ & - & - & & - & - & & 1.015 & 0.900 \\
\hline$\varepsilon\left(\Omega_{\mathrm{m}}\right)$ & 0.0226 & 0.0197 & & 0.0418 & 0.0400 & & 0.1485 & 0.1503 \\
$\varepsilon\left(H_{0}\right)$ & 0.0036 & 0.0028 & 0.0075 & 0.0050 & & 0.0139 & 0.0098 \\
$\varepsilon(w)$ & - & - & & 0.0737 & 0.0598 & & - & - \\
$\varepsilon\left(w_{0}\right)$ & - & - & & - & - & & 0.1436 & 0.1098 \\
\hline
\end{tabular}

Table 3. Constraint errors and accuracies for the cosmological parameters in the $\Lambda$ CDM, $w$ CDM and CPL models by using the simulated data of ET and CE. 

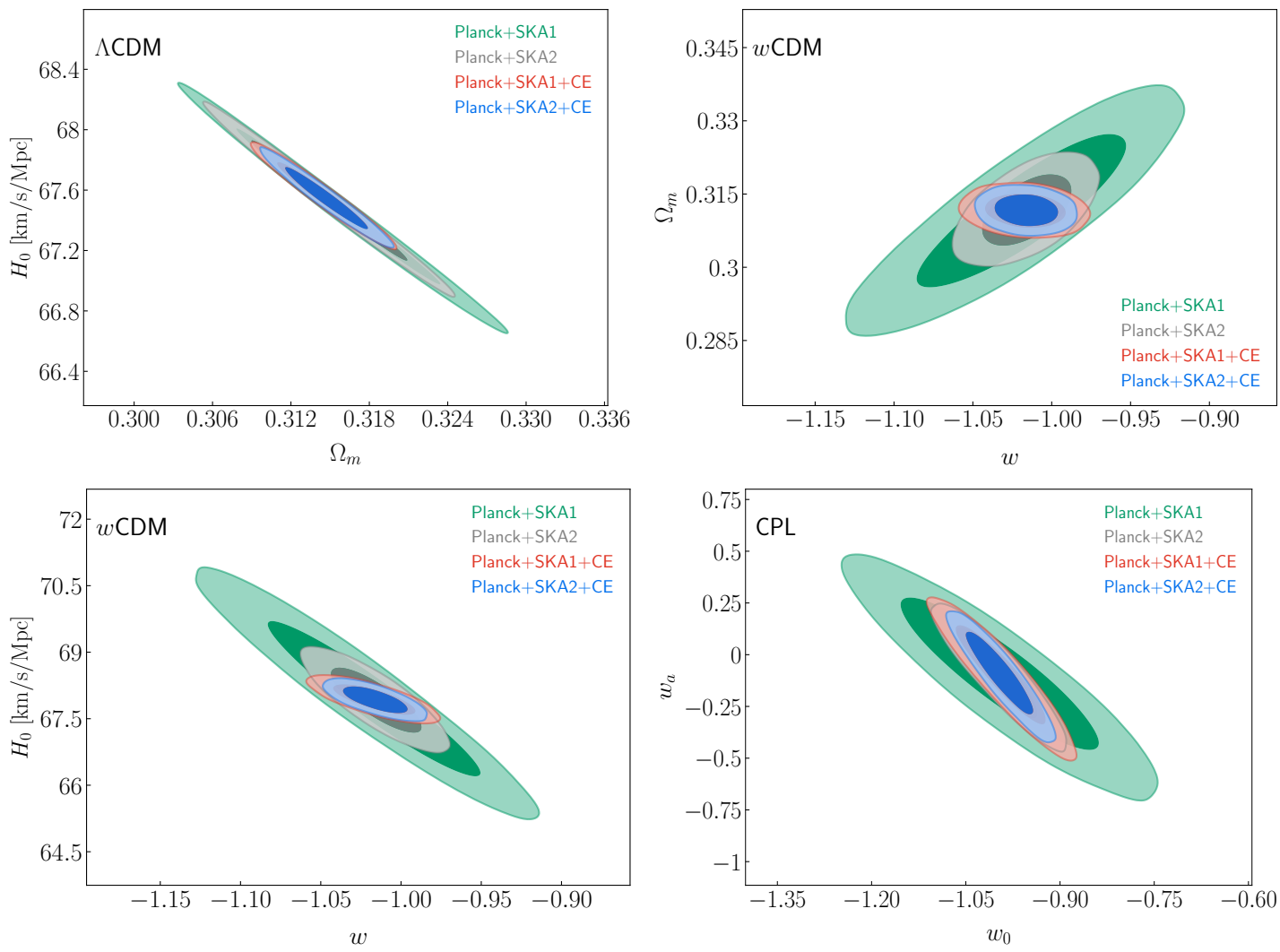

Figure 4. Constraints (68.3\% and $95.4 \%$ confidence level) on the $\Lambda$ CDM, $w$ CDM, and CPL models by using the Planck+SKA1, Planck+SKA2, Planck+SKA1+CE, Planck+SKA2+CE data combinations.

\begin{tabular}{ccccc}
\hline Data & Planck+SKA1 & Planck+SKA2 & Planck+SKA1+CE & Planck+SKA2+CE \\
\hline$\Omega_{\mathrm{m}}$ & $0.3158_{-0.0052}^{+0.0052}$ & $0.3149_{-0.0040}^{+0.0040}$ & $0.3144_{-0.0023}^{+0.0023}$ & $0.3147_{-0.0021}^{+0.0021}$ \\
$H_{0}$ & $67.48_{-0.34}^{+0.34}$ & $67.53_{-0.26}^{+0.26}$ & $67.56_{-0.15}^{+0.15}$ & $67.55_{-0.14}^{+0.14}$ \\
\hline$\sigma\left(\Omega_{\mathrm{m}}\right)$ & 0.0052 & 0.0040 & 0.0023 & 0.0021 \\
$\sigma\left(H_{0}\right)$ & 0.34 & 0.26 & 0.15 & 0.14 \\
\hline$\varepsilon\left(\Omega_{\mathrm{m}}\right)$ & 0.0165 & 0.0127 & 0.0073 & 0.0067 \\
$\varepsilon\left(H_{0}\right)$ & 0.0050 & 0.0039 & 0.0022 & 0.0021 \\
\hline
\end{tabular}

Table 4. The best-fit values of parameters, corresponding constraint errors and constraint accuracies within the $\Lambda \mathrm{CDM}$ model by using the Planck+SKA1, Planck+SKA2, Planck+SKA1+CE, and Planck+SKA2+CE data combinations. Here, $H_{0}$ is in units of $\mathrm{km} \mathrm{s}^{-1} \mathrm{Mpc}^{-1}$.

model, we find that the addition of CE mock data to the current optical observations (i.e., the CBS datasets) could break the degeneracy between the matter density and the Hubble constant, and further improve the constraint accuracies in the cosmological fit to a great 


\begin{tabular}{ccccc}
\hline Data & Planck+SKA1 & Planck+SKA2 & Planck+SKA1+CE & Planck+SKA2+CE \\
\hline$\Omega_{\mathrm{m}}$ & $0.312_{-0.010}^{+0.010}$ & $0.3117_{-0.0048}^{+0.0048}$ & $0.3116_{-0.0023}^{+0.0023}$ & $0.3117_{-0.0021}^{+0.0021}$ \\
$H_{0}$ & $68.00_{-1.20}^{+1.10}$ & $67.93_{-0.49}^{+0.49}$ & $67.94_{-0.22}^{+0.22}$ & $67.93_{-0.20}^{+0.20}$ \\
$w$ & $-1.019_{-0.041}^{+0.046}$ & $-1.016_{-0.019}^{+0.019}$ & $-1.017_{-0.017}^{+0.017}$ & $-1.016_{-0.013}^{+0.013}$ \\
\hline$\sigma\left(\Omega_{\mathrm{m}}\right)$ & 0.0100 & 0.0048 & 0.0023 & 0.0021 \\
$\sigma\left(H_{0}\right)$ & 1.15 & 0.49 & 0.22 & 0.20 \\
$\sigma(w)$ & 0.0435 & 0.0190 & 0.0170 & 0.0130 \\
\hline$\varepsilon\left(\Omega_{\mathrm{m}}\right)$ & 0.0321 & 0.0154 & 0.0074 & 0.0067 \\
$\varepsilon\left(H_{0}\right)$ & 0.0169 & 0.0072 & 0.0032 & 0.0029 \\
$\varepsilon(w)$ & 0.0427 & 0.0187 & 0.0167 & 0.0128 \\
\hline
\end{tabular}

Table 5. Same as table 4 , but for the $w$ CDM model (adding one more parameter, $w$ ).

\begin{tabular}{ccccc}
\hline Data & Planck+SKA1 & Planck+SKA2 & Planck+SKA1+CE & Planck+SKA2+CE \\
\hline$\Omega_{\mathrm{m}}$ & $0.312_{-0.016}^{+0.014}$ & $0.3108_{-0.0066}^{+0.0066}$ & $0.3121_{-0.0046}^{+0.0046}$ & $0.3118_{-0.0042}^{+0.0042}$ \\
$H_{0}$ & $68.00_{-1.60}^{+1.60}$ & $68.07_{-0.58}^{+0.58}$ & $67.92_{-0.39}^{+0.39}$ & $67.95_{-0.31}^{+0.31}$ \\
$w_{0}$ & $-0.99_{-0.10}^{+0.10}$ & $-0.999_{-0.044}^{+0.044}$ & $-0.992_{-0.048}^{+0.048}$ & $-0.995_{-0.035}^{+0.035}$ \\
$w_{a}$ & $-0.10_{-0.24}^{+0.24}$ & $-0.09_{-0.13}^{+0.16}$ & $-0.10_{-0.15}^{+0.17}$ & $-0.09_{-0.12}^{+0.14}$ \\
\hline$\sigma\left(\Omega_{\mathrm{m}}\right)$ & 0.0150 & 0.0066 & 0.0046 & 0.0042 \\
$\sigma\left(H_{0}\right)$ & 1.60 & 0.58 & 0.39 & 0.31 \\
$\sigma\left(w_{0}\right)$ & 0.100 & 0.044 & 0.048 & 0.035 \\
$\sigma\left(w_{a}\right)$ & 0.240 & 0.145 & 0.160 & 0.130 \\
\hline$\varepsilon\left(\Omega_{\mathrm{m}}\right)$ & 0.0481 & 0.0212 & 0.0147 & 0.0135 \\
$\varepsilon\left(H_{0}\right)$ & 0.0235 & 0.0085 & 0.0057 & 0.0046 \\
$\varepsilon\left(w_{0}\right)$ & 0.1010 & 0.0440 & 0.0484 & 0.0352 \\
\hline
\end{tabular}

Table 6. Same as table 4 , but for the CPL model (adding two more parameters, $w_{0}$ and $w_{a}$ ).

extent. Concretely, when adding the simulated CE data to the CBS datasets, the constraint precisions of $\Omega_{m}$ and $H_{0}$ in the $\Lambda$ CDM model could be improved from $1.59 \%$ and $0.49 \%$ to $0.70 \%$ and $0.21 \%$, respectively. Moreover, as shown in the planes of $w-H_{0}$ and $w-\Omega_{\mathrm{m}}$, we also find that the parameter degeneracy orientations of GW evidently differ from those of CBS data combination, which implies that the CE mock data can help to largely break 
the parameter degeneracies between these parameters in the $w \mathrm{CDM}$ model. With the help of CE mock data, the constraint precisions of $\Omega_{m}, H_{0}$ and $w$ can be improved from $2.47 \%$, $1.22 \%$, and $3.15 \%$ to $0.74 \%, 0.32 \%$, and $1.68 \%$, respectively. Furthermore, the constraint results on the CPL model are shown in the $w_{0}-w_{a}$ plane. We can apparently find that the constraining capability of CE is powerful as well, and the constraint accuracies of $\Omega_{m}$, $H_{0}$ and $w_{0}$ could be improved from $2.50 \%, 1.24 \%$, and $8.37 \%$ to $1.38 \%, 0.54 \%$, and $5.04 \%$, respectively, with the addition of $\mathrm{CE}$ mock data. What is supposed to be emphasized is that the central value of $w_{a}$ is around zero, and thus the absolute error for this parameter is more reliable for quantifying the improvement as the relative error would be impacted by its statistic fluctuations. For the parameter $w_{a}$, the absolute constraint error is improved from 0.305 to 0.160 . Therefore, we can conclude that the GW standard siren observations from $\mathrm{CE}$ will be able to significantly improve the cosmological parameter constraints in the near future.

\section{Some discussions}

\subsection{Comparison of CE and ET}

As a newly proposed third-generation GW detector, CE is a L-shaped single interferometer, with the angle between two arms equal to $90^{\circ}$ and the length of arms reaching an astonishing $40 \mathrm{~km}$. While the same-type ground-based GW detector ET consists of three identical interferometers with $10 \mathrm{~km}$ arms, with the angle between two arms equal to $60^{\circ}$, forming an equilateral triangle. Since both CE and ET belong to the third-generation groundbased GW detectors, it is necessary to make a comparison for the promotion effects on the parameter estimation from the GW standard siren observations of $\mathrm{CE}$ and $\mathrm{ET}$. In figure 1, we show the sensitivity curves of CE and ET. For the ET mock data, the simulation method is similar to that of $\mathrm{CE}$ as described in section 3.1. Then, we use the CE and ET mock data to constrain the three considered DE models separately. The constraint results are shown in figure 3 and summarized in table 3 .

From figure 3, we can clearly see that the constraint results from CE are slightly better than those from ET. Concretely, the constraint precisions of the parameters $\Omega_{m}$ and $H_{0}$ from $\mathrm{CE}$ are higher than those of ET by $1.1 \%-12.7 \%$ and $20.8 \%-33.3 \%$, respectively. As for the EoS parameters $w$ and $w_{0}$, the constraint precisions from CE are higher than those of ET by $18.7 \%$ and $22.6 \%$. For the parameter $w_{a}$, the constraint absolute error from CE is smaller than that of ET by $11.3 \%$. Thus, it can be seen that the constraining power of $\mathrm{CE}$ on the cosmological parameters maybe stronger than that of ET in the future.

\subsection{Synergy with SKA}

As an important non-optical cosmological probe besides the GW standard sirens, the HI $21 \mathrm{~cm}$ observation with the SKA will also play an important role in the future cosmological parameter estimation (see, e.g., refs. [34, 36-38]). Hence, it is also necessary to discuss the synergy of the GW standard siren observation from the CE with the $\mathrm{HI} 21 \mathrm{~cm}$ radio observation from the SKA. As a next-generation radio observatory, the SKA is currently under development, with the total collecting area being one square kilometer. Here, we consider the HI intensity mapping observations with the SKA phase 1 mid-frequency array (denoted as SKA1-MID), and the HI galaxy survey with SKA phase 2 (denoted as SKA2). They are both able to observe the large-scale structure at the redshift range of $0 \lesssim z \lesssim 3$ where DE dominates the evolution of the universe. 
We use the simulated data of the BAO measurements from the HI sky survey based on SKA1 and SKA2 in ref. [60]. For the SKA1-MID intensity mapping, the experimental specifications used in the forecast are given in table 2 of ref. [60]. For the SKA2 HI galaxy survey, the expected galaxy number counts and bias of HI galaxies used in the forecast can be found in table 1 of ref. [60]. The expected relative errors of $H(z)$ and $D_{A}(z)$ in the BAO measurements by the SKA, using the Fisher forecasting method, are given in figure 3 of ref. [60].

We employ the Planck+SKA1, Planck+SKA2, Planck+CE+SKA1, and Planck+CE+SKA2 data combinations to constrain the $\Lambda \mathrm{CDM}, w \mathrm{CDM}$, and CPL models and make the analysis. The constraint results are shown in figure 4 and summarized in tables 4-6. In figure 4, we show the two-dimensional posterior distribution contours for the cosmological parameters (i.e., $\Omega_{\mathrm{m}}, H_{0}, w, w_{0}$, and $w_{a}$ ) constrained at $68 \%$ and $95 \%$ C.L.. In tables $4-6$, we display the constraint errors and the constraint accuracies for the concerned parameters.

From figure 4 and tables $4-6$, we can apparently find that regardless of adding the $\mathrm{CE}$ mock data to either the Planck+SKA1 datasets or the Planck+SKA2 datasets, the constraint results could be significantly improved in all the considered DE models. For example, when adding the CE mock data to the Planck+SKA1 datasets, the constraints on the matter density $\Omega_{m}$ can be improved by $55.8 \%-77 \%$, and the constraints on the Hubble constant $H_{0}$ can be promoted by $55.9 \%-80.9 \%$. With respect to the parameters featuring the property of DE, the improvements are also evident, with the constraints on the parameter $w$ in the $w \mathrm{CDM}$ model promoted by $60.9 \%$, and the parameters of $w_{0}$ and $w_{a}$ in the CPL model improved by $52 \%$ and $33.3 \%$, respectively. Hence, we can conclude that the synergy between the GW standard siren observation from CE and the $21 \mathrm{~cm}$ emission observation from SKA would be extremely effective on the cosmological parameter estimation.

\section{Conclusion}

In this paper, we have investigated how the future GW standard sirens observation from $\mathrm{CE}$ impact the cosmological parameter estimation. For the conventional cosmological probes based on the EM observation, we use the latest CMB data from Planck 2018, the optical $\mathrm{BAO}$ measurements, and the SN observation of Pantheon compilation. We consider three typical cosmological DE models in this work, including the $\Lambda \mathrm{CDM}$ model, the $w \mathrm{CDM}$ model and the CPL model. In order to quantify the constraining ability of the additional GW data, we consider another two data combinations, namely CBS and CBS+GW, to constrain these DE models.

We find that the GW standard siren observation from the CE could tremendously improve the constraints on the cosmological parameters (i.e., $\Omega_{\mathrm{m}}, H_{0}, w, w_{0}$, and $w_{a}$ ). With the addition of CE in CBS datasets, the constraints on $\Omega_{m}$ can be improved by $44.9 \%-70.1 \%$, and the constraints on $H_{0}$ can be improved by $56.0 \%-73.5 \%$. For the constraints on those EoS parameters, the improvements are also evident, with the parameter $w$ in $w \mathrm{CDM}$ model promoted by $46.9 \%, w_{0}$ and $w_{a}$ in CPL model promoted by $39.8 \%$ and $47.5 \%$ respectively. What's more, we also find that degeneracies between several cosmological parameters, such as $\Omega_{m}$ and $H_{0}$ in $\Lambda$ CDM model, $\Omega_{m}$ and $w$ in $w$ CDM model as well as $w_{0}$ and $w_{a}$ in CPL model, could be significantly broken with the addition of CE mock data in the cosmological fit. Therefore, we conclude that the future GW standard siren from the CE could provide significant improvement in parameter estimation. 
In addition, we make a comparison for the parameter constraining capability of the GW standard siren observations from ET and CE. We find that the constraint results from CE are slightly better than those from ET. Furthermore, we discuss the synergy of the GW standard siren observation from the CE with the $\mathrm{HI} 21 \mathrm{~cm}$ radio waves observation from the SKA. We find that the synergy between the GW standard siren observation from $\mathrm{CE}$ and the $21 \mathrm{~cm}$ emission observation from SKA will be effective on the cosmological parameter estimation in the future.

\section{Acknowledgments}

We thank Jing-Zhao Qi and Hai-Li Li for helpful discussions. This work was supported by the National Natural Science Foundation of China (Grant Nos. 11975072, 11690021, 11875102, 11835009, 11973047, and 11633004), the CAS Strategic Priority Research Program (Grant No. XDA15020200), the Liaoning Revitalization Talents Program (Grant No. XLYC1905011), and the Fundamental Research Funds for the Central Universities (Grant No. N2005030).

\section{References}

[1] A. G. Riess et al. [Supernova Search Team], Astron. J. 116 (1998) 1009 doi:10.1086/300499 [astro-ph/9805201].

[2] S. Perlmutter et al. [Supernova Cosmology Project Collaboration], Astrophys. J. 517 (1999) 565 doi:10.1086/307221 [astro-ph/9812133].

[3] N. Aghanim et al. [Planck Collaboration], arXiv:1807.06209 [astro-ph.CO].

[4] M. E. Levi et al. [DESI Collaboration], arXiv:1907.10688 [astro-ph.IM].

[5] https://www.euclid-ec.org/

[6] https://www.lsst.org/

[7] B. P. Abbott et al. [LIGO Scientific and Virgo Collaborations], Phys. Rev. Lett. 119 (2017) no.16, 161101 doi:10.1103/PhysRevLett.119.161101 [arXiv:1710.05832 [gr-qc]].

[8] B. P. Abbott et al. [LIGO Scientific and Virgo and Fermi GBM and INTEGRAL and IceCube and IPN and Insight-Hxmt and ANTARES and Swift and Dark Energy Camera GW-EM and DES and DLT40 and GRAWITA and Fermi-LAT and ATCA and ASKAP and OzGrav and DWF (Deeper Wider Faster Program) and AST3 and CAASTRO and VINROUGE and MASTER and J-GEM and GROWTH and JAGWAR and CaltechNRAO and TTU-NRAO and NuSTAR and Pan-STARRS and KU and Nordic Optical Telescope and ePESSTO and GROND and Texas Tech University and TOROS and BOOTES and MWA and CALET and IKI-GW Follow-up and H.E.S.S. and LOFAR and LWA and HAWC and Pierre Auger and ALMA and Pi of Sky and DFN and ATLAS Telescopes and High Time Resolution Universe Survey and RIMAS and RATIR and SKA South Africa/MeerKAT Collaborations and AstroSat Cadmium Zinc Telluride Imager Team and AGILE Team and 1M2H Team and Las Cumbres Observatory Group and MAXI Team and TZAC Consortium and SALT Group and Euro VLBI Team and Chandra Team at McGill University], Astrophys. J. 848 (2017) no.2, L12 doi:10.3847/2041-8213/aa91c9 [arXiv:1710.05833 [astro-ph.HE]].

[9] B. P. Abbott et al. [LIGO Scientific and Virgo and 1M2H and Dark Energy Camera GW-E and DES and DLT40 and Las Cumbres Observatory and VINROUGE and MASTER Collaborations], Nature 551 (2017) no.7678, 85 doi:10.1038/nature24471 [arXiv:1710.05835 [astro-ph.CO]]. 
[10] M. Abernathy et al. [ET Science Team], Einstein gravitational wave Telescope conceptual design study, ET-0106C-10 (issue: 4); see the website: www.et-gw.eu/.

[11] B. P. Abbott et al. [LIGO Scientific Collaboration], Class. Quant. Grav. 34 (2017) no.4, 044001 doi:10.1088/1361-6382/aa51f4 [arXiv:1607.08697 [astro-ph.IM]].

[12] W. Zhao and L. Wen, Phys. Rev. D 97, no. 6, 064031 (2018) doi:10.1103/PhysRevD.97.064031 [arXiv:1710.05325 [astro-ph.CO]].

[13] W. Zhao, B. S. Wright and B. Li, JCAP 1810, 052 (2018) doi:10.1088/1475-7516/2018/10/052 [arXiv:1804.03066 [astro-ph.CO]].

[14] W. Zhao and L. Santos, JCAP 1911, 009 (2019) doi:10.1088/1475-7516/2019/11/009 [arXiv:1710.10055 [astro-ph.HE]].

[15] R. G. Cai and T. Yang, Phys. Rev. D 95 (2017) no.4, 044024 doi:10.1103/PhysRevD.95.044024 [arXiv:1608.08008 [astro-ph.CO]].

[16] H. L. Li, D. Z. He, J. F. Zhang and X. Zhang, arXiv:1908.03098 [astro-ph.CO].

[17] X. N. Zhang, L. F. Wang, J. F. Zhang and X. Zhang, Phys. Rev. D 99 (2019) no.6, 063510 doi:10.1103/PhysRevD.99.063510 [arXiv:1804.08379 [astro-ph.CO]].

[18] X. Zhang, Sci. China Phys. Mech. Astron. 62 (2019) 110431 doi:10.1007/s11433-019-9445-7 [arXiv:1905.11122 [astro-ph.CO]].

[19] J. F. Zhang, H. Y. Dong, J. Z. Qi and X. Zhang, arXiv:1906.07504 [astro-ph.CO].

[20] J. F. Zhang, M. Zhang, S. J. Jin, J. Z. Qi and X. Zhang, JCAP 1909 (2019) no.09, 068 doi:10.1088/1475-7516/2019/09/068 [arXiv:1907.03238 [astro-ph.CO]].

[21] L. F. Wang, X. N. Zhang, J. F. Zhang and X. Zhang, Phys. Lett. B 782 (2018) 87 doi:10.1016/j.physletb.2018.05.027 [arXiv:1802.04720 [astro-ph.CO]].

[22] LISA, https://lisa.nasa.gov/.

[23] J. Mei, C. Shao and Y. Wang, arXiv:1510.04754 [gr-qc].

[24] J. Luo et al. [TianQin Collaboration], Class. Quant. Grav. 33 (2016) no.3, 035010 doi:10.1088/0264-9381/33/3/035010 [arXiv:1512.02076 [astro-ph.IM]].

[25] X. C. Hu et al., Class. Quant. Grav. 35 (2018) no.9, 095008 doi:10.1088/1361-6382/aab52f [arXiv:1803.03368 [gr-qc]].

[26] H. T. Wang et al., Phys. Rev. D 100 (2019) no.4, 043003 doi:10.1103/PhysRevD.100.043003 [arXiv:1902.04423 [astro-ph.HE]].

[27] C. Shi et al., Phys. Rev. D 100 (2019) no.4, 044036 doi:10.1103/PhysRevD.100.044036 [arXiv:1902.08922 [gr-qc]].

[28] Y. L. Wu, Int. J. Mod. Phys. A 33 (2018) no.31, 1844014 doi:10.1142/S0217751X18440141 [arXiv:1805.10119 [physics.gen-ph]].

[29] W. H. Ruan, Z. K. Guo, R. G. Cai and Y. Z. Zhang, arXiv:1807.09495 [gr-qc].

[30] N. Tamanini, C. Caprini, E. Barausse, A. Sesana, A. Klein and A. Petiteau, JCAP 1604 (2016) 002 doi:10.1088/1475-7516/2016/04/002 [arXiv:1601.07112 [astro-ph.CO]].

[31] L. F. Wang, Z. W. Zhao, J. F. Zhang and X. Zhang, arXiv:1907.01838 [astro-ph.CO].

[32] Z. W. Zhao, L. F. Wang, J. F. Zhang and X. Zhang, arXiv:1912.11629 [astro-ph.CO].

[33] https://www.skatelescope.org/

[34] D. J. Bacon et al. [SKA Cosmology Science Working Group], Publ. Astron. Soc. Austral. 37 (2020) e007 doi:10.1017/pasa.2019.51 [arXiv:1811.02743 [astro-ph.CO]]. 
[35] Y. Xu, X. Wang and X. Chen, Astrophys. J. 798, no. 1, 40 (2015) doi:10.1088/0004-637X/798/1/40 [arXiv:1410.7794 [astro-ph.CO]].

[36] Y. Xu, J. Hamann and X. Chen, Phys. Rev. D 94, no. 12, 123518 (2016) doi:10.1103/PhysRevD.94.123518 [arXiv:1607.00817 [astro-ph.CO]].

[37] J. F. Zhang, L. Y. Gao, D. Z. He and X. Zhang, Phys. Lett. B 799 (2019) 135064 doi:10.1016/j.physletb.2019.135064 [arXiv:1908.03732 [astro-ph.CO]].

[38] J. F. Zhang, B. Wang and X. Zhang, arXiv:1907.00179 [astro-ph.CO].

[39] Y. Xu and X. Zhang, arXiv:2002.00572 [astro-ph.CO].

[40] Y. Y. Xu and X. Zhang, Eur. Phys. J. C 76 (2016) no.11, 588 doi:10.1140/epjc/s10052-016-4446-5 [arXiv:1607.06262 [astro-ph.CO]].

[41] B. S. Sathyaprakash and B. F. Schutz, Living Rev. Rel. 12 (2009) 2 doi:10.12942/lrr-2009-2 [arXiv:0903.0338 [gr-qc]].

[42] W. Zhao, C. Van Den Broeck, D. Baskaran and T. G. F. Li, Phys. Rev. D 83 (2011) 023005 doi:10.1103/PhysRevD.83.023005 [arXiv:1009.0206 [astro-ph.CO]].

[43] Tjonnie G. F. Li, Extracting Physics from Gravitational Waves, Springer Theses (2015) doi:10.1007/978-3-319-19273-4

[44] K. Liao, X. L. Fan, X. H. Ding, M. Biesiada and Z. H. Zhu, Nature Commun. 8 (2017) no.1, 1148 Erratum: [Nature Commun. 8 (2017) no.1, 2136] doi:10.1038/s41467-017-01152-9, 10.1038/s41467-017-02135-6 [arXiv:1703.04151 [astro-ph.CO]].

[45] A. A. Abdo et al. [Fermi-LAT and Fermi GBM Collaborations], Science 323 (2009) 1688. doi:10.1126/science.1169101

[46] E. Nakar, A. Gal-Yam and D. B. Fox, Astrophys. J. 650 (2006) 281 doi:10.1086/505855 [astro-ph/0511254].

[47] L. Rezzolla, B. Giacomazzo, L. Baiotti, J. Granot, C. Kouveliotou and M. A. Aloy, Astrophys. J. 732 (2011) L6 doi:10.1088/2041-8205/732/1/L6 [arXiv:1101.4298 [astro-ph.HE]].

[48] R. Schneider, V. Ferrari, S. Matarrese and S. F. Portegies Zwart, Mon. Not. Roy. Astron. Soc. 324 (2001) 797 doi:10.1046/j.1365-8711.2001.04217.x [astro-ph/0002055].

[49] C. Cutler and D. E. Holz, Phys. Rev. D 80 (2009) 104009 doi:10.1103/PhysRevD.80.104009 [arXiv:0906.3752 [astro-ph.CO]].

[50] https://cosmicexplorer.org/researchers.html.

[51] http://www.et-gw.eu/index.php/etsensitivities

[52] B. S. Sathyaprakash, B. F. Schutz and C. Van Den Broeck, Class. Quant. Grav. 27 (2010) 215006 doi:10.1088/0264-9381/27/21/215006 [arXiv:0906.4151 [astro-ph.CO]].

[53] D. Reitze et al., arXiv:1907.04833 [astro-ph.IM].

[54] L. Chen, Q. G. Huang and K. Wang, JCAP 1902 (2019) 028 doi:10.1088/1475-7516/2019/02/028 [arXiv:1808.05724 [astro-ph.CO]].

[55] F. Beutler et al., Mon. Not. Roy. Astron. Soc. 416 (2011) 3017 doi:10.1111/j.1365-2966.2011.19250.x [arXiv:1106.3366 [astro-ph.CO]].

[56] A. J. Ross, L. Samushia, C. Howlett, W. J. Percival, A. Burden and M. Manera, Mon. Not. Roy. Astron. Soc. 449 (2015) no.1, 835 doi:10.1093/mnras/stv154 [arXiv:1409.3242 [astro-ph.CO]].

[57] S. Alam et al. [BOSS Collaboration], Mon. Not. Roy. Astron. Soc. 470 (2017) no.3, 2617 doi:10.1093/mnras/stx721 [arXiv:1607.03155 [astro-ph.CO]]. 
[58] D. M. Scolnic et al., Astrophys. J. 859 (2018) no.2, 101 doi:10.3847/1538-4357/aab9bb [arXiv:1710.00845 [astro-ph.CO]].

[59] R. Braun, A. Bonaldi, T. Bourke, E. Keane and J. Wagg, arXiv:1912.12699 [astro-ph.IM].

[60] P. Bull, S. Camera, A. Raccanelli, C. Blake, P. G. Ferreira, M. G. Santos and D. J. Schwarz, PoS AASKA14 (2015) 024 [arXiv:1501.04088 [astro-ph.CO]]. 